dissection). The medial aspects of affected ureters are freed from surrounding tissues, brought to the midline, and secured with mattress sutures through only the ureteral wall and Waldeyer's sheath, to lengthen the submucosal ureteral tunnels. The authors stress that suturing close to the ureteral wall (instead of through the deep trigonal muscle) is crucial to the long-term success of laparoscopic trigonoplasty.

The authors performed 27 procedures in 24 girls and 3 boys (mean age 8.2 years, range 2-16 years), of whom 14 patients had bilateral and 13 had unilateral VUR. The mean operative time was $147 \mathrm{~min}$ (range 60-240 min), with blood loss $<50 \mathrm{ml}$. No patients required conversion to open surgery; the only intraoperative complication reported was peritoneal perforation, which occurred in four patients during creation of the extraperitoneal space (only one patient required suturing). The mean hospital stay was 2.7 days. Reflux resolved in 38 ureters (in 24 patients), and 25 patients (93\%) were infection-free without antibiotics at the last follow-up (range 4-19 months).

Simforoosh and colleagues note that extended follow-up of these patients is necessary to establish the long-term success rate of their procedure.

Original article Simforoosh N et al. (2007) Extraperitoneal laparoscopic trigonoplasty for treatment of vesicoureteral reflux: novel technique duplicating its open counterpart. J Urol 177: 321-324

\section{Excellent 5-year outcomes for laparoscopic partial nephrectomy}

Open partial nephrectomy (OPN) is the current standard of care for patients with small renal tumors, mainly because of the excellent oncologic and renal postsurgical outcomes seen at $\geq 10$ years. Laparoscopic partial nephrectomy (LPN) has similar short-term and medium-term outcomes to OPN, but long-term outcomes are, generally, not yet available.

Lane and Gill have now reported excellent 5-year outcomes for 56 of their patients (mean age 64 years, range 30-85 years) who underwent LPN for a single, localized renal mass (mean size $2.9 \mathrm{~cm}$, range $1.3-7.0 \mathrm{~cm}$ ). Most tumors (91\%) were discovered incidentally. A retrospective review of patient data revealed that eight patients died of unrelated causes during follow-up, but no patient died of cancer-related causes; overall and cancerspecific 5-year survival rates for the whole group were $86 \%$ and $100 \%$, respectively. Among the 37 patients (66\%) with renal cell carcinoma (RCC), no patient experienced distant recurrence and only one experienced local recurrence: by 4 years after LPN, this patient developed papillary RCC at a different site, which had progressed from an indeterminate $<1 \mathrm{~cm}$ lesion seen on preoperative imaging. The 5 -year recurrence-free survival in patients with RCC was $97.3 \%$. When abnormal renal function was defined as serum creatinine $\geq 1.5 \mathrm{mg} / \mathrm{dl}$, abnormal renal function was present in seven patients preoperatively and developed after LPN in another three patients (6\%).

Lane and Gill conclude that LPN is underused in the US, despite oncologic and renal functional outcomes that are comparable to those of OPN at 5 years after surgery.

Original article Lane BR and Gill IS (2007) 5-year outcomes of laparoscopic partial nephrectomy. J Urol 177: 70-74

\section{Pelvicol ${ }^{\circledR}$ implants reduce recurrence of anterior vaginal wall prolapse}

The risk of requiring surgery for pelvic-organ prolapse or urinary incontinence increases with age, and reoperations are not uncommon. Many different graft materials have been used to help reduce prolapse recurrence; Meschia and colleagues investigated the efficacy of porcine skin collagen implants (Pelvicol ${ }^{\circledR}$ CR Bard, Inc., Murray Hill, NJ) for preventing recurrent anterior vaginal wall prolapse.

The authors evaluated outcomes in 201 women (aged $\leq 80$ years) with anterior vaginal wall prolapse of stage II or above, who were randomly allocated to undergo primary anterior vaginal repair with $(n=98)$ or without $(n=103)$ Pelvicol $^{\circledR}$ reinforcement. Patients were followed up at 3, 6 and 12 months, and annually thereafter.

Most women were satisfied with their outcome 1 year after surgery (only $11 \%$ of women reported pelvic-organ prolapse symptoms). These patients' self-reported severity of prolapse symptoms decreased significantly from baseline to 1 year $(P<0.001)$. Anatomical anterior prolapse recurrence was reported by $7 \%$ 